\title{
Microwave ponderomotive forces in solid-state ionic plasmas*
}

\author{
J. H. Boosket,a) and R. F. Cooper \\ University of Wisconsin, Madison, Wisconsin 53706 \\ S. A. Freeman \\ Hewlett-Packard Corporation, Microwave Technology Division, Santa Rosa, California 95403 \\ K. I. Rybakov and V. E. Semenov \\ Institute of Applied Physics, Russian Academy of Sciences, Nizhny Novgorod, 603600, Russia
}

(Received 18 November 1997; accepted 2 January 1998)

\begin{abstract}
Numerous observations have been reported in the literature of enhanced mass transport and solid-state reaction rates during microwave heating of a variety of ceramic, glass, and polymer materials. An explanation for these controversial observations has eluded researchers for over a decade. This paper describes a series of recent experimental and theoretical investigations that provide an explanation for these intriguing observations in terms of ponderomotive forces acting on mobile ionic species. The ponderomotive phenomenon, like its conventional-plasma analog, can be described in the continuum model limit by combining the continuity, Poisson's, and transport equations. However, the solid-state plasma version typically manifests as a result of gradients in mobile charge mobility (e.g., near physical surfaces or interfaces), whereas the conventional plasma ponderomotive transport is typically a consequence of gradients in the radiation field intensity. Both cases can be captured in a single, general, mathematical articulation developed in terms of the mobile particle fluxes. (C) 1998 American Institute of Physics. [S1070-664X(98)91005-9]
\end{abstract}

\section{INTRODUCTION}

It is widely recognized that microwave electromagnetic radiation can be effectively used to heat a variety of dielectric materials, including food and plasmas, as well as some commercial successes in materials processing such as drying of wood and curing of rubber products. Continuing interest in commercializing a broader spectrum of microwave heating applications in materials processing is based on more volumetric heating, rapid switching control of the power source, and capacity for selective coupling in heterogenous media when compared with conventional heating.

Successful commercialization of additional materials' processing applications will require demonstration of unique materials' synthesis capabilities or unique improvements in product quality, compelling cost advantages, repeatable process reliability, and effective marketing. At the present time, however, there are still many gaps of fundamental knowledge of the precise microwave materials' interaction mechanisms. Such information is important for specification of optimal microwave heating strategies and optimal process equipment design.

One of the most interesting examples of the importance and need for fundamental knowledge advancement concerns growing evidence that microwave heating promotes enhanced materials reaction or processing rates in comparison with conventional furnace heating at comparable temperatures. ${ }^{1}$ Specific examples include microwave sintering of ceramics, ${ }^{2-5}$ microwave-driven radioactive tracer ion

\footnotetext{
*Paper oThaI2-2 Bull. Am. Phys. Soc. 42, 2005 (1997).

"Invited speaker.

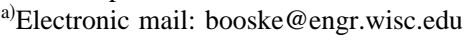

diffusion, ${ }^{6}$ microwave-driven ion-exchange reactions in glasses, ${ }^{7}$ microwave joining of ceramics, ${ }^{8}$ microwave (spinodal) decomposition of solid solutions, ${ }^{9}$ synthesis of metalcarbide powders, ${ }^{10}$ and promotion of organic imidization reactions. ${ }^{11}$

Understanding and explaining these observations has been complicated by difficulties in accurately characterizing the internal thermal state of a microwave-heated specimen. ${ }^{12}$ Also, some cases have been demonstrated to result from using different container designs for the microwave and conventionally heated experiments. ${ }^{13}$ Nevertheless, a significant number of recent experimental observations of microwaveinduced nonthermal reaction rate enhancements retain credibility, even after critical scrutiny.

As indicated in Fig. 1, reaction process rates, whether in solid, liquid, gas, or plasma phase, are determined by reactant transport rates (mixing), reactant collision rates (achieving intimate proximity of reacting species), and the probability of reaction per near-encounter (collision) of reacting particles. It is especially true in solid-state processes (which are the focus of this paper) that many process rates are transport limited (diffusion), due to the dense-packing which prohibits convective mixing. Therefore any microwavematerials' interactions that enhance mobile particle solidstate transport are likely to also enhance process rates for those solids.

Recently, it has been discovered that microwave field strengths typical of some materials' processing conditions exert an additional driving force for mobile ion transport in solids. ${ }^{14}$ Careful comparisons with a recent theoretical model ${ }^{15}$ have confirmed that this phenomenon is the result of microwave ponderomotive forces. ${ }^{16}$ Numerical analyses ${ }^{17}$ 


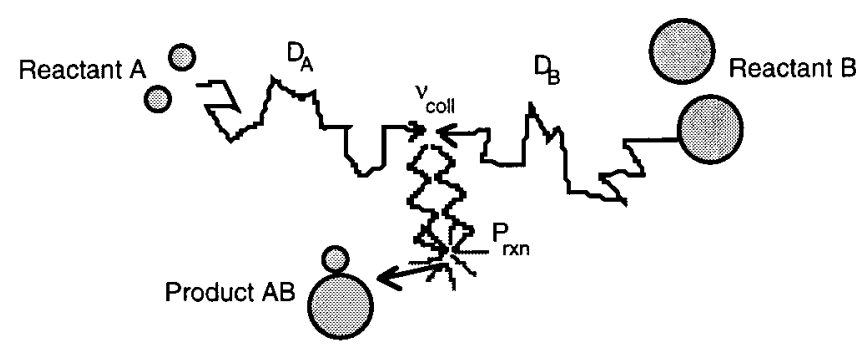

FIG. 1. Illustration of event sequence for all reactions (solid, liquid, or gas phase): reactant transport, reactant collisions, reaction (with probability $\left.P_{\text {rxn }}\right)$. Modification of reaction rates is accomplished by effecting changes in any or all of these steps, but especially the rate-limiting step.

have established that these ponderomotive forces can, indeed, be strong enough to compete with conventional driving forces in various examples of heat processing of ceramics and glasses.

In the remainder of this paper, we describe the mechanism and review the sequence of theoretical, experimental, and numerical studies identifying, verifying, and characterizing the phenomenon. The ponderomotive phenomenon, like its conventional-plasma analog, can be described in the continuum model limit by combining the continuity, Poisson's, and transport equations. However, the solid-state plasma version typically manifests as a result of gradients in charge mobility (e.g., near surfaces or interfaces), whereas the conventional plasma ponderomotive transport is typically a consequence of gradients in the radiation field intensity. It is illustrated how both cases can be captured in a single mathematical articulation developed in terms of the mobile particle fluxes.

\section{DISCOVERY, IDENTIFICATION, AND VERIFICATION OF THE PHENOMENON AND MECHANISM}

The first experimental evidence for strong microwave field effects on the transport of mobile ions in solid-state media was obtained from measurements of ionic currents in halide salt crystals exposed to brief pulses of microwave energy. ${ }^{18,19}$ These experiments were designed to evaluate whether strong microwave fields could enhance solid-state ion transport by increasing ionic mobility. The experimental configuration is schematically illustrated in Fig. 2 and described in detail in Ref. 20. An external bias voltage was

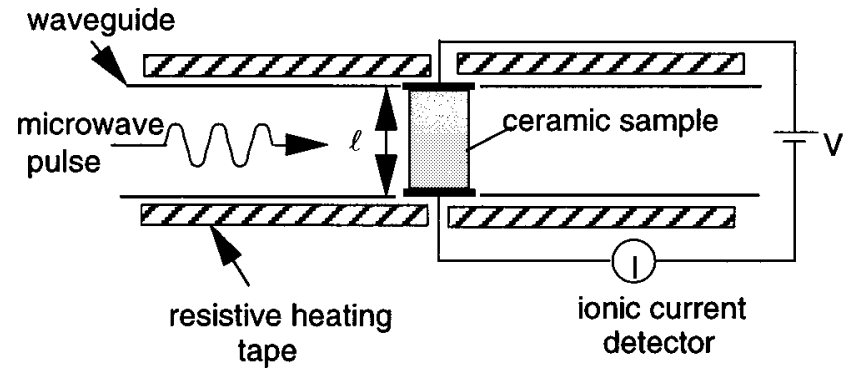

FIG. 2. Schematic illustration of the configuration used to observe microwave field effects on ionic currents in halide salt crystals.
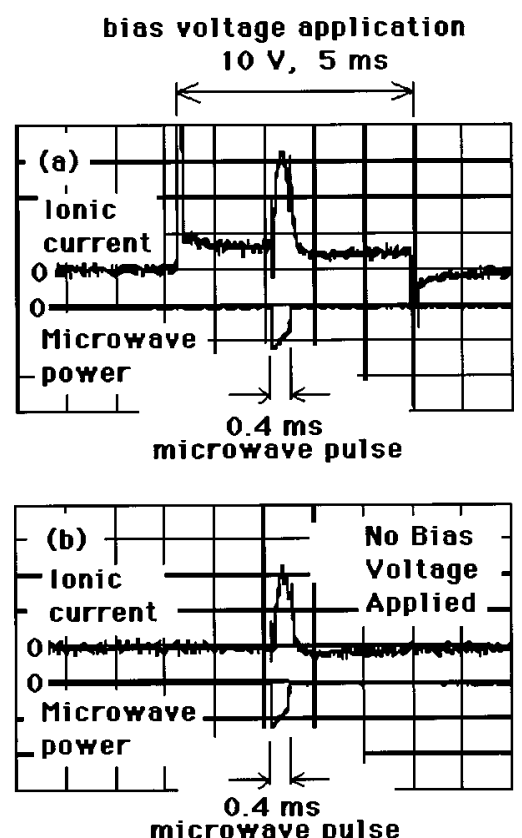

FIG. 3. Oscilloscope traces showing the effect of microwave irradiation on ionic currents in a $\mathrm{NaCl}$ crystal at $150{ }^{\circ} \mathrm{C}$. In both frames, the upper trace is the ionic current and the lower trace is the microwave power. Horizontal scale is $1 \mathrm{~ms} /$ div. (a) ionic current results from application of a $10 \mathrm{~V}$ bias across the crystal and is enhanced during microwave irradiation $(\sim 2 \mathrm{~kW})$; (b) no bias voltage is applied, yet still an ionic current pulse is observed during microwave irradiation.

applied to the crystal, inducing an ionic current, while the specimen's temperature was controlled by resistive heating tapes. While the external bias voltage was applied, the specimen was irradiated by a short pulse of microwave energy, and an increase in the ionic current was observed to result. After establishing that the observation was definitely an increase in ionic current and not electrode effects (e.g., Schottky-diode-like behavior) or electronic current, the question to resolve was whether the increased current was a consequence of increased ionic mobility (or conductivity) or due to an additional, microwave-induced driving force for ionic diffusion. As illustrated in Fig. 3, this question was resolved in favor of the latter mechanism by varying the applied bias voltage. During this process, it was observed that the additional increment of current during the microwave pulse remained unaffected by the applied bias voltage, and only depended on the intensity of the microwave field. It was also shown that the microwave-induced part of the ionic current scaled in proportion to the square of the microwave electric field intensity, suggesting a nonlinear interaction. ${ }^{14,19}$

Around the same time, a theoretical model was proposed $^{15}$ that suggested microwave fields could enhance ionic transport in solid-state "ionic plasmas" through the influence of a ponderomotive force interaction. The fact that a high-frequency electromagnetic field influences the timeaveraged motion of charged particles is well-known in conventional plasma physics. In particular, the slowly varying influence of a spatially nonuniform, high-frequency electromagnetic field acting on a particle is referred to as a ponderomotive force (PMF). ${ }^{21}$ In the case of these solid-state ionic plasmas, the theoretical model predicted that a similar 
nonlinear effect could manifest near physical surfaces or interfaces, should scale with the square of the electric field, and should be sensitive to the surface conditions of the irradiated material. Both of these latter qualitative features of the theoretical model were consistent with the experimental observations. ${ }^{14}$

The theoretical analysis involves a second-order perturbation treatment of the transport, continuity, and Poisson's equations, i.e.,

$$
\begin{aligned}
& \mathbf{J}_{i}=-D_{i} \nabla n_{i}+\frac{D_{i} n_{i}}{k T} q_{i} \mathbf{E}, \\
& \frac{\partial n_{i}}{\partial t}=-\nabla \cdot \mathbf{J}_{i}, \\
& \nabla \cdot \mathbf{E}=\frac{1}{\epsilon} \sum_{i} q_{i}\left(n_{i}-n_{i}^{(0)}\right),
\end{aligned}
$$

where the subscript $i$ refers to the mobile charge species, $\mathbf{J}$ is the particle flux, $q$ is the species charge, $D$ is the diffusion coefficient, $n$ is the concentration, $k$ is Boltzmann's constant, $T$ is the temperature, and $\mathbf{E}$ is the electric field vector.

The basic idea is that a high-frequency (HF) harmonic electric field drives a high-frequency flux of charge mobile species within the medium. In the bulk of the medium, the fluxes are spatially uniform. Near the surface, however, the discontinuity of the medium results in a harmonic oscillation of the ionic concentration, as it alternately accumulates and depletes during a HF cycle. These oscillations in the nearsurface concentrations are in phase (or nearly so) with the HF electric field oscillations. As seen from Eq. (1), any such in-phase oscillations of electric field and mobile charge concentration multiply to produce a rectified, quasistationary flux of charge carriers after averaging over a HF cycle.

Expanding $\mathbf{J}, n$, and $\mathbf{E}$ in Eqs. (1)-(3) to second order

$$
X(t) \approx X^{(0)}+X^{(1)}+X^{(2)},
$$

where $X^{(0)}$ represents static (equilibrium) solutions, $X^{(1)}$ represents HF fluctuation terms, and $X^{(2)}$ represents the periodaveraged, quasistationary components, yields the quasistationary ionic flux

$$
\begin{aligned}
J_{i}^{(2)} \approx & -D_{i} \nabla n_{i}^{(2)}+\frac{D_{i} n_{i}^{(2)}}{k T} q_{i} \mathbf{E}^{(2)} \\
& +\frac{D_{i} n_{i}^{(0)}}{k T}\left\langle\left(\frac{n_{i}^{(1)}}{n_{i}^{(0)}}\right) q_{i} \mathbf{E}^{(1)}\right),
\end{aligned}
$$

where the angular brackets indicate a fast-time-average over the period of the HF field oscillations. The microwaveinduced ponderomotive driving force is identified as the time-averaged piece of the last term of Eq. (5)

$$
\mathrm{PMF}=\left\langle\left(\frac{n^{(1)}}{n^{(0)}}\right) q E^{(1)}\right\rangle .
$$

Generally, the HF charge density perturbation $n^{(1)}$ will be localized near the physical interface or surface, within the smaller of either the Debye-Hückel radius $\lambda=\sqrt{D \epsilon / 2 \sigma}$ $=\sqrt{\epsilon k T / 2 n_{i} q_{i}^{2}}$ (solid-state equivalent of the Debye length) or the characteristic diffusion length, $\ell=\sqrt{D / \omega}$ (at microwave frequencies, $\omega$, one can expect the latter distance to apply for mobile ion charge density perturbations). For a onedimensional case, the HF charge perturbation will accompany a perturbation of the HF electric field such that

$$
n^{(1)}=\frac{\epsilon}{q} \frac{\partial E^{(1)}}{\partial x} .
$$

Substituting Eq. (7) into (6) reveals the quadratic dependence of the PMF on the electric field intensity:

$$
\mathrm{PMF} \propto\left\langle\frac{\epsilon}{2} \frac{\partial\left|E^{(1)}\right|^{2}}{\partial x}\right\rangle .
$$

Based, then, on qualitative similarities between predicted features of the model ${ }^{15}$ and the experimental data, ${ }^{14}$ a collaboration was undertaken to refine both the model and the experiments to permit a more detailed comparison. The eventual outcome of that effort was a confirmation that the microwave-enhanced ionic transport observed in the experiments was the result of the PMF originally proposed in Ref. 15. The principal achievement of the collaborative study was to demonstrate agreement between the theory and the experimental data regarding sophisticated details of transient features in the time-dependent ionic current induced by the microwave pulses. ${ }^{16}$

\section{NUMERICAL SIMULATIONS AND THEORY REFINEMENTS}

In order to examine the physics of the solid-state PMF in greater detail as well as to evaluate its relevance to conditions present during microwave processing of materials, numerical simulations were investigated for a solid-state ionic plasma modeled by the three relevant equations (1)-(3). ${ }^{17}$ The simulations examined in detail the illustrative case of mobile ion response to microwave frequency electric fields near the surface of a sodium chloride crystal (fields polarized normal to the surface). In ionic single crystals, it is often convenient to describe the diffusion of mobile ions in terms of the complementary transport of charged point defects (vacancies) and that is the approach used in these simulations.

Figure 4 illustrates the fact that the HF perturbation is localized near the surface of the crystal [see the discussion between Eqs. (6) and (7)]. However, by modifying the local quasistatic ionic (defect) concentration gradient (or electrochemical potential) near such a surface, the PMF's influence propagates with time into the bulk of the medium. This is illustrated in Fig. 5, which plots the charged mobile $\mathrm{Cl}$ (vacancy) ion concentration as a function of time and distance from the crystal surface. In this particular instance, the mobile charged species are continuously drawn from the bulk to the surface, resulting in a density depletion that reaches deeply into the crystal bulk with increasing time. As expected, the simulations confirmed the theoretical prediction that the PMF should drive mass transport: i.e., transport in the same direction for both positively and negatively charged mobile ionic species.

Owing to the fact that $\mathrm{NaCl}$ is a well-characterized material, accurate quantitative predictions were possible. This 


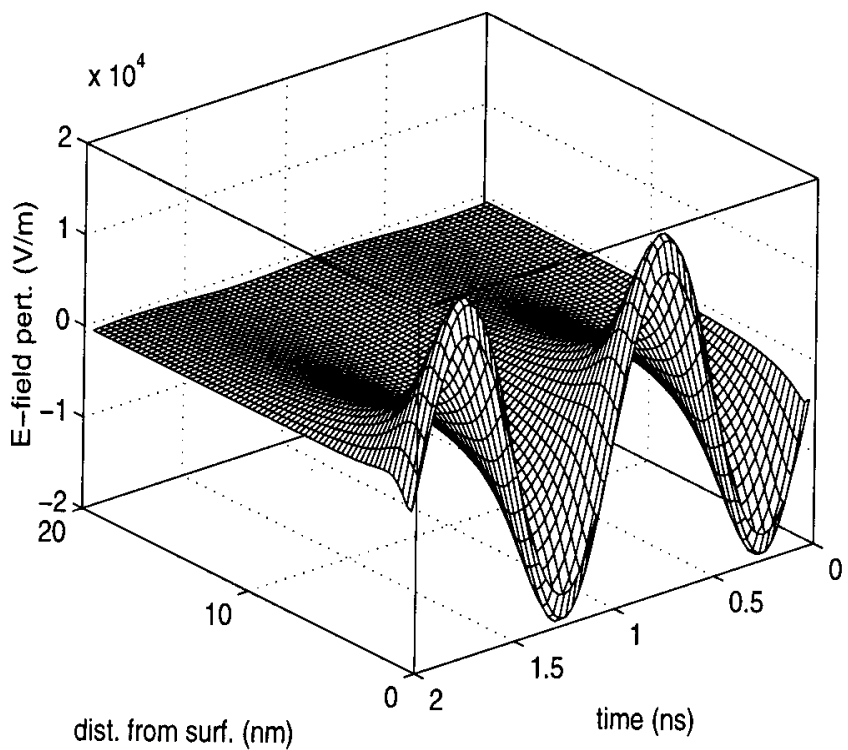

FIG. 4. High-frequency $(1 \mathrm{GHz})$ electric field perturbation as a function of time and distance from the crystal surface.

effort established that the magnitude of the PMF for microwave field intensities typical of materials processing conditions can equal or exceed the magnitudes of other competitive transport driving forces that would be present in the absence of the microwave field. ${ }^{22}$ Thus it was established that the microwave PMF is capable of explaining enhanced solid-state reactions by significantly enhancing reactant transport in ionic solids. It may seem remarkable that these solid-state ponderomotive forces can have such a significant phenomenological effect for relatively low radiation pressures (in comparison, say, with mechanical stresses that may be present or can be applied). This apparent anomaly is understood by recognizing that the solid-state ponderomotive

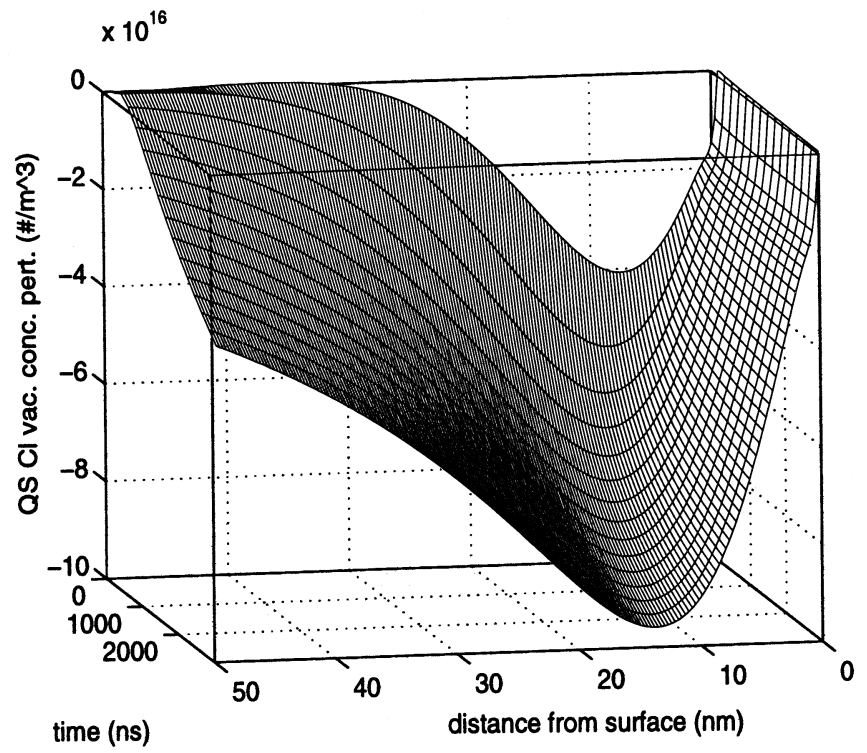

FIG. 5. Time- and space-dependent quasistatic perturbation to the ionic species (chlorine vacancy) concentration under the influence of microwavefrequency, near-surface ponderomotive forces.

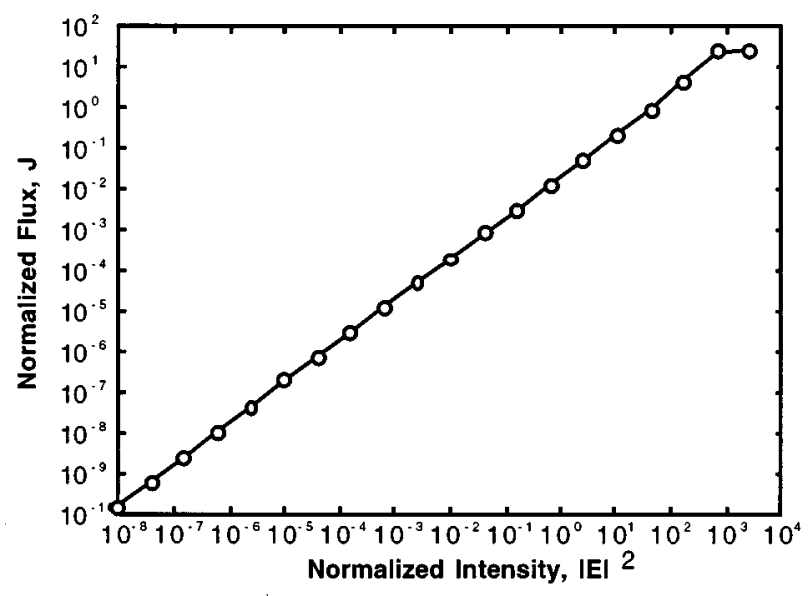

FIG. 6. Normalized ponderomotive-driven ionic flux versus normalized radiation field pressure. The PMF-driven flux scales linearly with the normalized radiation pressure to electric field intensities far in excess of either the expected validity of the perturbation-theory-derived expression or experimentally realizable values.

pressure is concentrated on the mobile species, whereas a mechanical pressure is distributed throughout all ions or atoms in the structure. ${ }^{23}$

Another interesting observation obtainable from the simulations is the limit of the perturbation model for predicting the PMF [cf. Eq. (6) or (8)]. For example, to examine the range over which the PMF-driven flux scales linearly with the radiation pressure (i.e., the square of the microwave electric field) we have plotted in Fig. 6 the simulation-predicted normalized flux versus a normalized expression for the PMF. The flux has been normalized to the value one might expect for an extremely severe concentration gradient

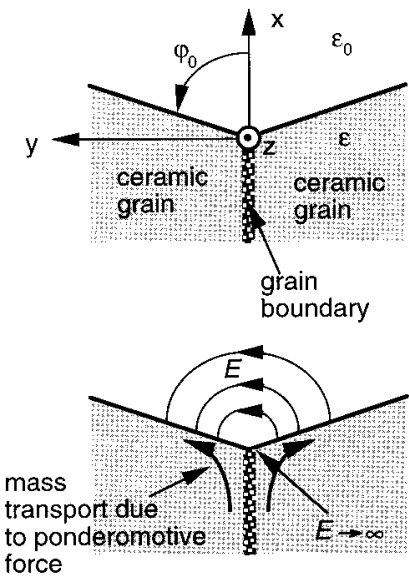

(a)

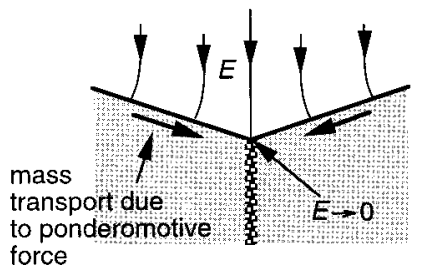

(b)

(c)

FIG. 7. Illustration of the geometry and electric field solutions near the grain boundary of two ceramic grains undergoing being sintered by microwave energy. 


$$
J_{\text {norm }}=\frac{J}{J_{\max }}, \quad J_{\max }=D|\nabla n|_{\max } \sim D \frac{n_{0}}{\ell}=n_{0} \sqrt{D \omega}
$$

while the normalized PMF has been characterized in terms of the normalized radiation pressure:

$$
\mathrm{PMF} \propto\left|E_{\text {norm }}\right|^{2} \equiv\left|\frac{q \widetilde{E}_{H F} \ell}{k T}\right|^{2}=\frac{D q^{2}}{\omega(k T)^{2}}\left|\widetilde{E}_{H F}\right|^{2} .
$$

As indicated in Fig. 6, the PMF-driven flux scales linearly with the normalized radiation pressure to electric field intensities far in excess of experimentally realizable values. ${ }^{24}$ Hence the expression derived for the PMF from the perturbation analysis is much more generally valid than one would conclude on the basis of perturbation expansion assumptions.

Other recent additions to the theory concern the role of electric fields polarized tangential to the solid surfaces ${ }^{23}$ as well as mesoscopic field enhancements near pores in ceramic powder compacts, prior to high-temperature sintering. For example, Calame et al. have recently established by numerical simulation ${ }^{25}$ that the intensity of the electric field is enhanced in spherical neck regions between two spherical powder grains undergoing microwave sintering. Due to faceting, ${ }^{5}$ a more appropriate geometry for description of realistic sintering experiments is two flat grain surfaces forming a dihedral angle, as illustrated in Fig. 7. The electric field enhancement in this geometry can be described by solving the corresponding quasielectrostatics problem. Choosing cylindrical coordinates with the $z$ axis directed along a grain boundary edge [cf. Fig. 7(a)], the following expression for the electrostatic potential $\Phi$ can be obtained:

$$
\Phi=\left\{\begin{array}{c}
A r^{\alpha} \frac{\sin \alpha \varphi}{\sin \alpha \varphi_{0}}+B r^{\beta} \frac{\cos \beta \varphi}{\cos \beta \varphi_{0}}, \quad|\varphi| \leqslant \varphi_{0} \\
A r^{\alpha} \frac{\sin \alpha(\pi-\varphi)}{\sin \alpha\left(\pi-\varphi_{0}\right)}+B r^{\beta} \frac{\cos \beta(\pi-\varphi)}{\cos \beta\left(\pi-\varphi_{0}\right)}, \\
\varphi_{0}<|\varphi| \leqslant \pi
\end{array}\right.
$$

where $\varphi$ is the azimuthal angle, the sector $\varphi_{0}<|\varphi|<\pi$ is occupied by the solid (with dielectric constant $\epsilon$ ), whereas for $|\varphi|<\varphi_{0}$ there is vacuum. In Eqs. (11), $A$ and $B$ are arbitrary constants, and $\alpha$ and $\beta$ are the smallest positive roots of the equations

$$
\begin{aligned}
& \epsilon \tan \alpha \varphi_{0}=-\tan \alpha\left(\pi-\varphi_{0}\right), \\
& \tan \beta \varphi_{0}=-\epsilon \tan \beta\left(\pi-\varphi_{0}\right) .
\end{aligned}
$$

The first terms on the right of Eqs. (11) correspond to the antisymmetric mode [Fig. 7(b)], in which the electric field vector $\mathbf{E}=-\nabla \Phi$ points normal to the symmetry plane $(\varphi$ $=0, \varphi=\pi)$. The second terms correspond to the symmetric mode [Fig. 7(c)], in which the electric field vector in the symmetry plane is parallel to that plane.

Since generally, for sintering of ceramic micropowders $\varphi_{0}<\pi / 2$, it follows from Eqs. (12) that $\alpha<1$ and $\beta>1$. Therefore in the antisymmetric mode the electric field strength increases without limit, $E \propto r^{\alpha-1}$ when approaching the grain boundary edge (i.e., $r \rightarrow 0$ ), whereas in the symmetric mode $E \rightarrow 0$ as $r \rightarrow 0$. It should be noted that for large values of $\epsilon$, such that $\epsilon \tan \left(\pi / 2 \cdot \varphi_{0} /\left(\pi-\varphi_{0}\right)\right) \gg 1$ it can be approximately obtained that $\alpha \approx \pi /\left[2\left(\pi-\varphi_{0}\right)\right]$. Analysis of such solutions shows that the net ponderomotive action of the enhanced (antisymmetric) electric field results in mass flow along the grain surfaces and away from the interior of the intergrain boundary, as indicated in Fig. 7(b). In this fashion, the "amplified" ponderomotive action effectively extracts atoms from the intergrain boundary and facilitates the shrinkage of closed pores in ceramics undergoing microwave processing.

Insofar as the theoretical description is based on continuum equations, the ponderomotive phenomena can be expected to manifest in any solid medium with mobile ions, whether crystalline or amorphous. ${ }^{7}$ Moreover, solids conventionally regarded as covalent may still possess sufficient fractional ionization to manifest ponderomotive-force-enhanced mass transport during microwave heating. For example, metal carbides or nitrides are generally regarded as nonionic. However, such compounds are not completely covalent, ${ }^{26}$ and possess adequate fractional ionization (of order one electron charge out of four per mobile ion or defect) to be sensitive to ponderomotive forces. This may explain, for example, dramatically enhanced reaction rates in solid-state synthesis of $\mathrm{TiC}^{10}$

\section{GENERAL THEORY DISCUSSION}

Following Ref. 27, in this section, we compare and contrast the solid-state ionic plasma PMF with the more conventionally known plasma ponderomotive force. In general, the latter force is quadratic in the field strength and depends on having a nonuniform field. However, a precise definition of the ponderomotive force can only be given within a specific mathematical model of the system. Consequently, several different physical quantities may be called the ponderomotive force. We consider the three most important examples.

The Euler hydrodynamics model makes use of the following basic equations:

$$
\begin{aligned}
& \frac{\partial n}{\partial t}+\nabla \cdot(n \mathbf{v})=0, \\
& m\left[\frac{\partial \mathbf{v}}{\partial t}+(\mathbf{v} \cdot \nabla) \mathbf{v}\right]=q \mathbf{E}+q[\mathbf{v} \times \mathbf{B}]-\nu \mathbf{v},
\end{aligned}
$$

where $\mathbf{v}$ is the hydrodynamic velocity, $m$ is the mass of the particles, $\mathbf{E}$ and $\mathbf{B}$ are the electric and magnetic field vectors, and $\nu$ is the friction coefficient or collision frequency. Defining the PMF (per unit volume) within this framework as the total averaged force exerted by the electromagnetic field,

$$
\mathbf{f}_{1}=m\langle n\rangle\left[\frac{\partial\langle\mathbf{v}\rangle}{\partial t}+(\langle\mathbf{v}\rangle \cdot \nabla)\langle\mathbf{v}\rangle+\nu\langle\mathbf{v}\rangle\right],
$$

where the angular brackets denote time averaging. Using this definition and solving Eqs. (13), $\mathbf{f}_{1}$ may be obtained as

$$
\mathbf{f}_{1}=-\frac{\omega_{p}^{2}}{\omega^{2}+\nu^{2}} \nabla\left\langle\frac{\epsilon \mathbf{E}^{2}}{2}\right\rangle+\frac{\omega_{p}^{2}}{\omega^{2}+\nu^{2}} \nu\langle\epsilon \mathbf{E} \times \mathbf{B}\rangle,
$$

where $\omega_{p}=\sqrt{n q^{2} / m \epsilon}$ is the conventional plasma frequency.

The single particle model proceeds from the equation of motion: 


$$
m \frac{\partial^{2} \mathbf{r}}{\partial t^{2}}=q \mathbf{E}(\mathbf{r}, t)+q\left[\frac{\partial \mathbf{r}}{\partial t} \times \mathbf{B}(\mathbf{r}, t)\right]-\nu(\mathbf{r}) \frac{\partial \mathbf{r}}{\partial t} .
$$

Within this approach, the ponderomotive force is defined as

$$
\mathbf{f}_{2}=m\langle n\rangle\left[\left\langle\frac{\partial^{2} \mathbf{r}}{\partial t^{2}}\right\rangle+\nu\left\langle\frac{\partial \mathbf{r}}{\partial t}\right\rangle\right] .
$$

To compare with the previous model, $\mathbf{f}_{2}$ can be obtained from Eq. (16) in the form:

$$
\begin{aligned}
\mathbf{f}_{2}= & \mathbf{f}_{1}+\frac{\omega_{p}^{2}}{\omega^{2}+\nu^{2}} \frac{\nu}{\omega^{2}}\left\langle(\epsilon \mathbf{E} \cdot \nabla) \frac{\partial \mathbf{E}}{\partial t}\right\rangle \\
& -\frac{\omega_{p}^{2}}{\omega^{2}+\nu^{2}}\left\langle\frac{\partial \mathbf{E}}{\partial t}\left(\epsilon \mathbf{E} \cdot \nabla \frac{\nu(\mathbf{r})}{\omega^{2}}\right)\right\rangle .
\end{aligned}
$$

As defined, $\mathbf{f}_{1}$ determines the dynamics of a timeaveraged field of velocities, whereas $\mathbf{f}_{2}$ determines the dynamics of motion of single particles. In general, $\langle\mathbf{v}\rangle$ $\neq\langle\partial \mathbf{r} / \partial t\rangle$ since in the former case the averaging is performed at a given point in space, whereas in the latter instance the averaging is done for a given particle along its trajectory. As known in plasma physics, in the $\nu=0$ case the PMF's defined via the two approaches $\left(\mathbf{f}_{1}\right.$ and $\left.\mathbf{f}_{2}\right)$ are equal. However, for $\nu \neq 0, \mathbf{f}_{1} \neq \mathbf{f}_{2}$, as seen from Eq. (18). In order to correctly describe the mass transport flux (which is a quantity of most interest for the microwave processing of materials application), one should use $\mathbf{f}_{2}$. For a sufficiently high value of $\nu$, it follows from Eq. (18) that $m\langle n\rangle\langle\partial \mathbf{r} / \partial t\rangle$ $=\mathbf{f}_{2} / \nu$. In comparison, the quantity $m\langle n\rangle\langle\mathbf{v}\rangle=\mathbf{f}_{1} / \nu$ does not fully describe the mass transport, since the mass transport flux also includes the term $m\langle(n-\langle n\rangle)(\mathbf{v}-\langle\mathbf{v}\rangle)\rangle$ which is not accounted for in $\mathbf{f}_{1}$. Viewed another way, the second term of the right-hand side of Eq. (18) particularly distinguishes $\mathbf{f}_{2}$ from $\mathbf{f}_{1}$ for the solid-state ionic plasma application. High-frequency charge density perturbations near abrupt physical interfaces [Eq. (6)] give rise to nonuniform high-frequency field perturbations [Eq. (7)], giving this distinguishing term a nonzero value.

To accurately describe the mass transport within the frame of a hydrodynamic approach, the hydrodynamic equations (13) should be formulated for the particle flux variable $\mathbf{J}=n \mathbf{v}$ instead of the hydrodynamic velocity $\mathbf{v}$ :

$$
\begin{aligned}
& \frac{\partial n}{\partial t}+\nabla \cdot \mathbf{J}=0, \\
& m\left\{\frac{\partial \mathbf{J}}{\partial t}+\left[(\mathbf{J} \cdot \nabla) \frac{\mathbf{J}}{n}+\frac{\mathbf{J}}{n}(\nabla \cdot \mathbf{J})\right]\right\}=q n \mathbf{E}+q[\mathbf{J} \times \mathbf{B}]-\nu \mathbf{J} .
\end{aligned}
$$

Consistent with the flux hydrodynamics model, the PMF is defined as

$$
\mathbf{f}_{3}=m\left\{\frac{\partial\langle\mathbf{J}\rangle}{\partial t}+\left[(\langle\mathbf{J}\rangle \cdot \nabla) \frac{\langle\mathbf{J}\rangle}{\langle n\rangle}+\frac{\langle\mathbf{J}\rangle}{\langle n\rangle}(\nabla \cdot\langle\mathbf{J}\rangle)\right]+\nu\langle\mathbf{J}\rangle\right\}
$$

and solving Eqs. (19) yields

$$
\begin{aligned}
\mathbf{f}_{3}= & \mathbf{f}_{2}-\frac{\nu}{\omega^{2}}\left\langle\left(\frac{\omega_{p}^{2}}{\omega^{2}+\nu^{2}} \frac{\partial \epsilon \mathbf{E}}{\partial t}\right) \cdot \nabla \mathbf{E}\right. \\
& \left.+\mathbf{E} \nabla \cdot\left(\frac{\omega_{p}^{2}}{\omega^{2}+\nu^{2}} \frac{\partial \epsilon \mathbf{E}}{\partial t}\right)\right) .
\end{aligned}
$$

In general, this last expression differs from both of the previous two. However, if the electric field has linear polarization, i.e.,

$$
\mathbf{E}=\mathbf{A}(\mathbf{r}) \exp [i \varphi(\mathbf{r})] \exp (i \omega t)+\text { c.c., } \quad \mathbf{A}=\mathbf{A}^{*},
$$

then the last term in Eq. (21) is zero and $\mathbf{f}_{3}=\mathbf{f}_{2}$. A circularly polarized field would cause rotation of particles. In the case of nonuniform plasma, this may result in additional rotational components in the flux, which are accounted for by $\mathbf{f}_{3}$ but not $\mathbf{f}_{2}$. Such rotational components of flux can be macroscopically detectable even when the radius of revolution of each particle is microscopic. Each rotating particle remains on its own closed circular trajectory with zero net averaged displacement. However, since the concentration of such particles is nonuniform, there may be a nonzero averaged macroscopic rotational flux in this situation. Nevertheless, the flux lines of this macroscopic flow will always be closed (i.e., $\nabla \cdot\langle\mathbf{J}\rangle=0$ ) and hence it is not of interest to the sort of mass transport flux important to the microwave processing application which is the subject of this paper. Therefore from the point of view of describing mass transport fluxes in solidstate ionic plasmas which are important for microwave materials' processing applications, $\mathbf{f}_{3}$ and $\mathbf{f}_{2}$ yield equivalent results. When the local electric field has a linear polarization (but is nonuniform), then both the last term in Eq. (21) and the very last term in Eq. (18) are zero, and

$$
\mathbf{f}_{3}=\mathbf{f}_{2}=\mathbf{f}_{1}+\frac{\omega_{p}^{2}}{\omega^{2}+\nu^{2}} \frac{\nu}{\omega^{2}}\left\langle(\epsilon \mathbf{E} \cdot \nabla) \frac{\partial \mathbf{E}}{\partial t}\right\rangle .
$$

It should also be noted that according to Eq. (18) (i.e., the third term), in the case of nonuniform collisionality, a nonzero PMF (either $\mathbf{f}_{2}$ or $\mathbf{f}_{3}$ ) can exist even if the electric field is uniform, although the Euler hydrodynamics model would predict no PMF under such conditions. This latter situation, which requires a local field polarization different than linear, specifically can apply to the solid-state ionic plasma conditions present during experimental observations of microwave-enhanced solid-state transport and reactions. In this regard, it is useful to note that even if the polarization of the external field is linear, the local field polarization can be elliptical due to a complex permittivity (i.e., associated with dissipation).

For solid-state applications the following limiting case is of special interest: $\nu \gg \omega_{p} \gg \omega$. In solid-state terms, this corresponds to the following condition, which is generally satisfied in ionic solids in the temperature and frequency ranges used in microwave processing technologies: $\omega \tau \ll d / \lambda \ll 1$, where $\tau$ is the time interval between atom hops, $d$ is interatomic distance, and $\lambda$ is the Debye-Hückel radius for the ionic plasma. As shown in Ref. 23, in the free quasielectrostatic limit where $\nabla \times \mathbf{E} \approx 0, \quad \nabla \cdot\left(\epsilon_{p} \mathbf{E}\right) \approx 0$ and $\epsilon_{p} \equiv 1$ 
$-\omega_{p}^{2} / \omega(\omega-i \nu)$, the solid-state plasma PMF takes an especially simple form, derived from the flux hydrodynamics approach,

$$
\mathbf{f}_{3} \approx\langle\rho \mathbf{E}\rangle,
$$

where $\rho=(\nabla \cdot \mathbf{E}) / 4 \pi$ is the density of the field-induced space charge (note that although $\mathbf{f}_{2}$ would not yield this form, it would give the same mass transport, since $\nabla \cdot \mathbf{f}_{2}=\nabla \cdot \mathbf{f}_{3}$ ). Equation (24) is actually the same as Eq. (6) in Sec. II.

A summary comparison of various approaches to the definition of the PMF suggests that while all of them are valid theoretical formulations, some of the approaches are more convenient than others for addressing specific problems. For example, microwave processing of solid materials is mostly dependent upon the mass transport phenomena which are responsible for both the process kinetics and final product properties. To describe the solid-state ionic plasma dynamics for this and similar applications, the flux hydrodynamics model and the single particle approach appear to be equally appropriate for defining the PMF since they enable one to compute the mass fluxes with no additional terms. The Euler hydrodynamics model, on the other hand, requires the ad hoc addition of the term $m\langle(n-\langle n\rangle)(\mathbf{v}-\langle\mathbf{v}\rangle)\rangle$ in order to describe these mass fluxes, as discussed below Eq. (18). Of all three approaches, the flux hydrodynamics model is considered preferable for solid-state problems with strong spatial nonuniformities on the surfaces, since it allows for efficient treatment of diffusion phenomena.

\section{CONCLUSIONS AND SUMMARY}

It has been demonstrated through theoretical analyses, experimental measurements, and numerical simulations that high-frequency electromagnetic fields exert ponderomotive forces on mobile ions in solids so as to enhance solid-state ionic diffusion. The ponderomotive phenomenon, like its conventional-plasma analog, can be described in the continuum model limit by combining the continuity, Poisson's, and transport equations. However, the solid-state plasma version typically manifests as a result of gradients in mobile charge mobility (e.g., near physical surfaces or interfaces), whereas the conventional plasma ponderomotive transport is typically a consequence of gradients in the radiation field intensity. It has been shown that both cases are most efficiently included in a common mathematical hydrodynamics model articulated in terms of the particle fluxes.

As a practical matter, it has been shown that the magnitude of the solid-state ionic ponderomotive force is sufficiently strong for realistic field intensities to explain experimentally observed enhancements of mass transport and solid-state reaction rates during microwave heating of ceramic or glass materials. Opportunities therefore exist for reduced processing times or temperatures or improved mate- rials' properties through the choice of microwave heating instead conventional furnace heating. Identifying conditions for which these advantages of microwave heating may be reliably realized requires further research.

\section{ACKNOWLEDGMENTS}

The authors acknowledge the encouragement and helpful comments of many colleagues. This work was supported in part by the National Science Foundation (Grant Nos. ECS9057675, MSM-8657164, and CTW-9526035), the National Aeronautics and Space Administration's Graduate Student Researcher Program, the U.S. Electric Power Research Institute, the Wisconsin Alumni Research Foundation, and the Russian Basic Science Foundation (Grant No. 97-02-17252).

${ }^{1}$ S. J. Rothman, Mater. Res. Soc. Symp. Proc. 347, 9 (1994).

${ }^{2}$ Y. V. Bykov, A. E. Eremeev, and V. V. Holoptsev, Mater. Res. Soc. Symp. Proc. 347, 585 (1994).

${ }^{3}$ R. Wroe and A. T. Rowley, J. Mater. Sci. 31, 2019 (1996).

${ }^{4}$ D. Dadon, L. P. Martin, M. Rosen, A. Birman, D. Gershon, J. P. Calame, B. Levush, and Y. Carmel, J. Mater. Syn. Proc. 4, 95 (1996).

${ }^{5}$ M. Willert-Porada, Ceram. Trans. 80, 153 (1997).

${ }^{6}$ M. A. Janney and H. D. Kimrey, Mater. Res. Soc. Symp. Proc. 189, 215 (1990).

${ }^{7}$ Z. Fathi, D. E. Clark, and R. Hutcheon, Mater. Res. Soc. Symp. Proc. 269, 347 (1992).

${ }^{8}$ D. Palaith, R. Silberglitt, C. C. M. Wu, R. Kleiner, and E. Libelo, Am. Ceram. Soc. Bull. 68, 1601 (1989).

${ }^{9}$ M. Willert-Porada, Mater. Res. Soc. Symp. Proc. 430, 403 (1996).

${ }^{10}$ J. G. P. Binner, N. A. Hassine, and T. E. Cross, J. Mater. Sci. 30, 5389 (1995)

${ }^{11}$ D. A. Lewis, J. D. Summers, T. C. Ward, and J. E. McGrath, J. Polym. Sci., Polym. Chem. Ed. 30, 1647 (1992).

${ }^{12}$ D. J. Grellinger and M. A. Janney, Ceram. Trans. 36, 529 (1993).

${ }^{13}$ K. C. Westaway and R. N. Gedye, J. Microwave Power Electromagn. Energy 30, 219 (1995).

${ }^{14}$ S. A. Freeman, J. H. Booske, and R. F. Cooper, Phys. Rev. Lett. 74, 2042 (1995).

${ }^{15}$ K. I. Rybakov and V. E. Semenov, Phys. Rev. B 49, 64 (1994).

${ }^{16}$ K. I. Rybakov, V. E. Semenov, S. A. Freeman, J. H. Booske, and R. F. Cooper, Phys. Rev. B 55, 3559 (1997)

${ }^{17}$ S. A. Freeman, Ph.D. thesis, University of Wisconsin, 1996.

${ }^{18}$ S. A. Freeman, J. H. Booske, R. F. Cooper, B. Meng, J. Kieffer, and B. J. Reardon, Ceram. Trans. 36, 213 (1993).

${ }^{19}$ S. Freeman, J. Booske, R. Cooper, and B. Meng, Mater. Res. Soc. Symp. Proc. 347, 479 (1994).

${ }^{20}$ S. A. Freeman, J. H. Booske, and R. F. Cooper, Rev. Sci. Instrum. 66, 3606 (1995)

${ }^{21}$ A. G. Litvak, in Reviews of Plasma Physics, edited by M. A. Leontovich (Consultants Bureau, New York, 1986), Vol. 10, p. 294.

${ }^{22}$ J. H. Booske, R. F. Cooper, and S. A. Freeman, J. Mater. Res. Innov. 1, 77 (1997).

${ }^{23}$ K. I. Rybakov and V. E. Semenov, Phys. Rev. B 52, 3030 (1995).

${ }^{24}$ For example, for a typical case of $\ell-1 \mathrm{~nm}$ and $k T / q \sim 0.1 \mathrm{eV}, E_{\text {norm }} \sim 1$ corresponds to $E \sim 10^{8} \mathrm{~V} / \mathrm{m}$.

${ }^{25}$ J. P. Calame, K. I. Rybakov, Y. Carmel, and D. Gershon, Ceram. Trans. 80, 135 (1997).

${ }^{26}$ W. Schmickler and J. W. Schultze, Ber. Bunsenges. Phys. Chem. 96, 760 (1992).

${ }^{27}$ K. I. Rybakov and V. E. Semenov, in Strong Microwaves in Plasmas, edited by A. G. Litvak (Nizhny Novgorod University Press, Nizhny Novgorod, 1997), Vol. 1, pp. 374-379. 